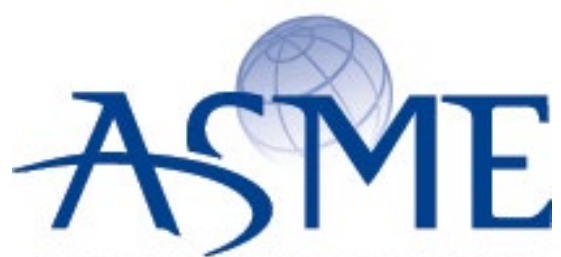

SETTING THE STANDARD

\section{American Society of Mechanical Engineers}

ASME Accepted Manuscript Repository

Institutional Repository Cover Sheet

I

IMark

King

First

Last

Muscle-tendon unit parameter estimation of a hill-type musculoskeletal model based on experimentally obtained subject-specific torque profiles

ASME Paper Title:

Authors:

F. Heinen1, S.N. Sørensen2, M.A. King3, M. Lewis4, M.E. Lund5, J. Rasmussen2, M. de Zee1

ASME Journal Title: The Journal of Biomechanical Engineering

Volume/Issue

141(6):61005

ASME Digital Collection URL: http://biomechanical.asmedigitalcollection.asme.org/article.aspx?articleid=2730405

DOI: $\quad 10.1115 / 1.4043356$

*VOR (version of record)
Date of Publication (VOR* Online) Apr $25^{\text {th }}$ 2019 


\title{
Muscle-tendon unit parameter estimation of a Hill-type musculoskeletal model based on experimentally obtained subject-specific torque profiles
}

\author{
F. Heinen ${ }^{1}$, S.N. Sørensen², M.A. King ${ }^{3}$, M. Lewis ${ }^{4}$, M.E. Lund ${ }^{5}$, J. Rasmussen², M. de Zee ${ }^{1}$ \\ ${ }^{1}$ Sport Sciences, Department of Health Science and Technology, Aalborg University, Denmark \\ ${ }^{2}$ Department of Materials and Production, Aalborg University, Denmark \\ ${ }^{3}$ School of Sport, Exercise and Health Sciences, Loughborough University, United Kingdom \\ 4 School of Science and Technology, Nottingham Trent University, United Kingdom \\ 5 AnyBody Technology A/S, Denmark
}

\section{ABSTRACT}

The aim of this study was to generate a subject-specific musculoskeletal muscle model, based on isometric and isovelocity measurements of the whole lower extremity. A twostep optimisation procedure is presented for optimising the muscle-tendon parameters for isometric and isovelocity joint torque profiles. A significant improvement in the prediction of joint torque profiles for both the solely isometric and a combined isometric and dynamic method of optimization when compared to the standard scaling method of The AnyBody Modeling System was observed. Depending on the specific purpose of the model, it may be worth considering whether the isometric-only would be sufficient, or the additional dynamic data are required for the combined approach.

Keywords: subject-specific model, muscle-tendon parameters, hill-muscle model, optimisation

\section{INTRODUCTION}

Internal joint and muscle forces can be very difficult or even impossible to measure in vivo and it might be difficult to acquire the appropriate ethical approval. Hence, musculoskeletal models may be used to estimate these forces in a noninvasive manner. However, invasive measurements are required in order to evaluate the predictive capability of these models. Several musculoskeletal models have been presented in the literature [1, 2] in various fields of application [3-5]. One of these fields is sports biomechanics, where models are used to optimize [6], characterize or analyze $[3,7]$ specific sport-related movements and determine the internal forces.

Musculoskeletal models are typically based on scalable generic models assuming a Hill-type muscle model [8, 9]. The Hill-type muscle model contains a contractile element (CE), a series elastic element (SE) and a parallel elastic element $(\mathrm{PE})$, characterizing the force-length-velocity relation of the muscle-tendon unit. Predictions using the Hill-type muscle model have been shown to be sensitive towards most its parameters, but in particular the optimal fiber length $\left(\mathrm{L}_{\mathrm{M}}^{\mathrm{o}}\right)$, tendon slack length $\left(\mathrm{L}_{\mathrm{t} 0}\right)$ and physiological cross sectional area [10, 11]. These muscletendon parameters (MTP) do not depend linearly on skeletal scaling [12] but are of a complex nature.

Several different MTP scaling methods have been presented in the literature $[13,14]$. Winby et al. [14] established the importance of preserving the muscle's operating range when anthropometrically scaling a generic model by comparing different scaling methods. Van Campen et al. [15] presented a method to maintain the generic force-producing capability of the scaled muscle model. These reported methods are very applicable for general analysis purposes but, when dealing with participants who most likely deviate in their strength characteristics from the general population, such as patients or elite athletes, the model should be adapted accordingly. Isokinetic dynamometers have been used widely to measure the isometric and dynamic strength of a participant in a standardized and controlled 
manner. Torque measurements collected on a dynamometer have likewise been widely used to generate subject-specific, torque-driven models and been shown to be a viable method to predict the crucial parameters in sport movements [16-19]. However, since musculoskeletal models comprise individual muscles rather than a net joint torque profile, additional steps have to be included.

Garner and Pandy [20] presented a method to adjust the isometric strength of the arm muscles according to experimentally obtained net isometric joint torques based on a mathematical optimisation procedure. However, this study only implemented isometric measurements and did not include the dynamic contraction capabilities of the muscles, which have been shown to vary amongst participants [21]; this is especially important when dealing with maximal high-velocity sport performances that approach the limits of human physical capability. Wu et al. [22] presented a method combining isometric and isokinetic experimental joint torque data of the glenohumeral joint in a two stage optimization procedure. However, this study was limited to the number of joints implemented and the amount of experimental joint torque information as well as only implementing monoarticular muscles. Moreover, d'Souza et al. [23] developed a multiple linear approximation law based on segment masses, age and gender and improved the strength prediction capability for knee extension and elbow flexion. However, the correlations between parameters that are measurable externally and the strength of an individual remains weak, even for nonathletic test subjects.

So far the presented studies have been limited to experimental joint torque data from a single joint and hence are often limited in practical use. Furthermore, studies determine muscle-tendon parameters of the lower limb have not determined these of a whole lower limb based on experimental measurements but have been limited to single joint measurements or simulated data.

The aim of this study was therefore to develop a method capable of generating a musculoskeletal model with subject-specific MTP based on maximal voluntary isometric as well as isovelocity torque measurements of a particular athlete, and to determine subject-specific MTP of the entire lower extremity.

\section{METHODS}

Experiment

One male long distance runner (height: $1.85 \mathrm{~m}$, weight: $66.5 \mathrm{~kg}$ ) was included in this study. In accordance with the local ethical review committee of Loughborough University, United Kingdom, a series of isometric and isovelocity experiments were conducted for the ankle, knee and hip (flexors/extensors) of the dominant leg using a Contrex multi-joint isokinetic dynamometer (CMV AG, Switzerland) (Figure 1A-C). The measurements comprised a familiarization session and three testing sessions on four separate days, to ensure maximal performance by the participant and to avoid fatigue influencing the measurements. A similar experimental protocol as reported by [19] was adopted. Seven evenly spaced isometric measurements were performed for each joint movement throughout the participant's joint range of motion (ROM). Six (ankle and hip) and eight (knee) isovelocity measurements from $50 \%$ s and increasing with increments of $50 \%$ were performed for both concentric and eccentric contractions. The dynamometer torque-angle-velocity data were sampled at $510 \mathrm{~Hz}$. The participant was firmly secured to the seat by straps and alignment of the dynamometer and joint center-of-rotation was made during submaximal loading. During the isometric contractions, the joint angle was measured using an electrogoniometer (Biometrics Ltd, United Kingdom) and a hand-held goniometer to avoid 
errors due to soft tissue deformation. The participant performed two maximum voluntary isometric contractions at each joint angle and three consecutive isovelocity measurements were collected for three repetitions of a concentric-eccentric protocol, repeated for all joint velocities. Rest periods were provided between the different angles, velocity and contraction.
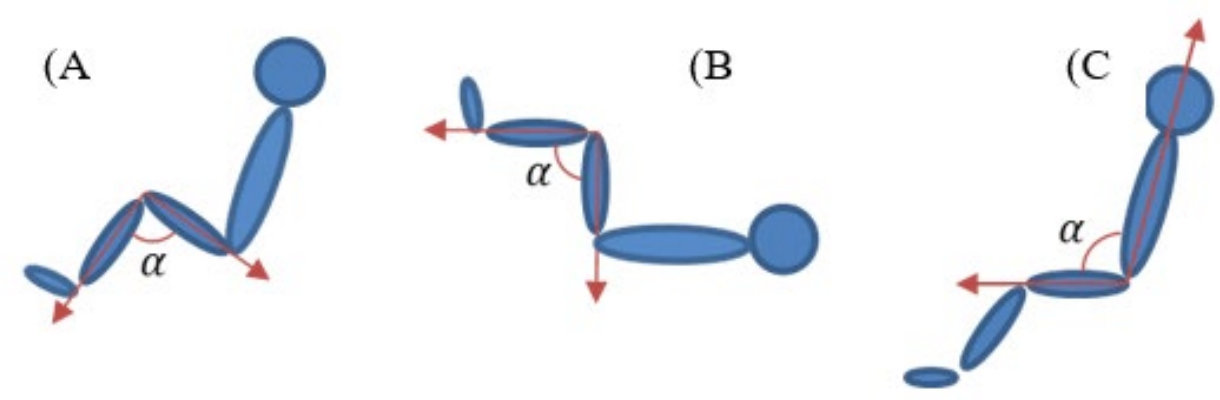

Figure 1. Experimental body position reference joint angles: The knee was fixed at $90^{\circ}$ flexion during ankle (A) and hip (B) measurements. The hip was fixed at $105^{\circ}$ flexion during knee (C) measurements.

\section{Data processing}

All torque measurements were corrected throughout the joint ROM for weight of the limb and passive torques, using the dynamometer built in software. The torque, angle and velocity data from the dynamometer and goniometer were filtered using a zero lag $12 \mathrm{~Hz}$, low pass, fourth-order Butterworth filter. The isometric trial which recorded the largest torque at each joint angle was selected for further analysis. At each joint velocity the trial selected was also that which recorded the largest torque at any point during the ROM. All torque measurements were selected with due consideration for avoiding measurement artefacts associated with the dynamometer function by eliminating periods of high acceleration or velocity overshoot. Velocity overshoot was identified as described by Schwartz et al. [24].

\section{Musculoskeletal model setup}

The lower extremity model based upon the TLEMsafe 2.0 data set [25] was applied in Anybody Modeling System TM version 6.1.0. The Hill-type muscle-tendon model implementation in Anybody Modeling System (AMS) is based on the assumptions presented by Daxner [26]:

The force-length $(\mathrm{FI})$ relation of the CE in AMS is based on the work presented by Otten [27], Daxner [26] and Gföhler et al. [28].

$F_{l}\left(l_{C E}\right)=e^{-\left(\frac{\left(\epsilon_{F}+1\right)^{\beta_{F}-1}}{\omega_{F}}\right)^{\rho_{F}}}$ where $\epsilon_{F}=\frac{l_{C E}-l_{F}^{o p t}}{l_{F}^{o p t}}$

Where $l_{C E}$ and $l_{F}^{o p t}$ represent the length of the CE and the optimal length of CE, respectively. The parameters $\beta_{F}, \omega_{F}$ and $\rho_{F}$ are set to the values presented by Kaufman et al. [29]. 
The force-velocity (Fv) relation in AMS is derived from the work of Daxner [26] and Gföhler et al. [28], defined as:

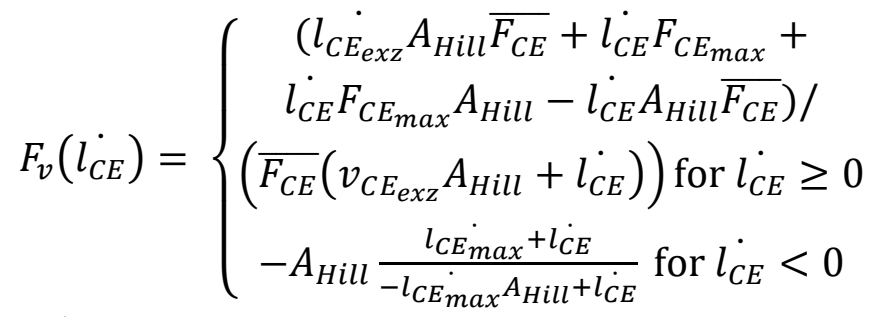

Where:

$l_{C E_{\text {max }}}=k_{1} \overline{l_{f}}+k_{2} \overline{l_{f}} C_{\text {fast }}$

$l_{C E_{\text {exz }}}=l_{C E_{\max }} \frac{F_{\max }-\bar{F}}{\bar{F}}$

Here $F_{\text {max }} \cong 1.3 \overline{F_{C E}}$ indicating the maximal force in an eccentric action, $A_{\text {Hill }}=0.1+$ $0.4 C_{\text {fast }}, k_{1}=0\left[s^{-1}\right], k_{2}=10\left[s^{-1}\right] . \overline{l_{f}}$ is the isometric tetanic fiber length and the subscript exz indicates the eccentric contraction.

The notations $\left(^{\cdot}\right)$ and $\left(^{-}\right)$denote the velocity state and tetanic state of the given elements, respectively. This muscle-tendon model has been used in several simulation studies and validated against experimental data [30, 31]. Some of the parameters in equations I through IV have a direct translation to the AMS AnyScript language, which has been indicated in Table 1.

Table 1. Translation of algorithm variables in equation I to IV to corresponding AnyBody script variables

\begin{tabular}{cc}
\hline Algorithm variables & Related AnyBody script variables \\
\hline$l_{F}^{\text {opt }}$ & $\mathrm{Lf0}$ \\
$C_{\text {fast }}$ & Fcfast \\
$k_{1}$ & $\mathrm{k} 1$ \\
$k_{2}$ & $\mathrm{k} 2$ \\
$\overline{F_{C E}}$ & $\mathrm{~F} 0$ \\
\hline
\end{tabular}

A series of musculoskeletal models were developed to compute the net joint torques of the model under similar circumstances as for the experiment. Muscle recruitment was based on the $\mathrm{min} / \mathrm{max}$ criterion, which is a reasonable assumption since the aim was to model maximal performance of the subject, who was instructed to perform so during the measurements. Furthermore, it has previously been shown to be viable when modelling maximum effort [32]. The model was scaled based on anthropometric measurements and the length-mass-fat scaling law [33].

In the following, a two-fold optimisation procedure will be presented; the first (isom-opt) concerns the isometric force-length relation and the related MTP. The second (dyn-opt) concerns the force-velocity relation and the related parameters and builds on top of the isom_opt results (Figure 2). The optimisation was divided into these two consecutive stages based on the nature of the experimental data, where isovelocity measurements are prone to more noise and motion artefacts than isometric measurements, and hence the isometric measurements were prioritised [34]. Furthermore, due to the experimental and computational time-consumption associated with the isovelocity part of the process, it seems relevant to compare the 
quality of results obtained solely from isometric data and isom-opt with results requiring isovelocity data and dyn-opt as well.
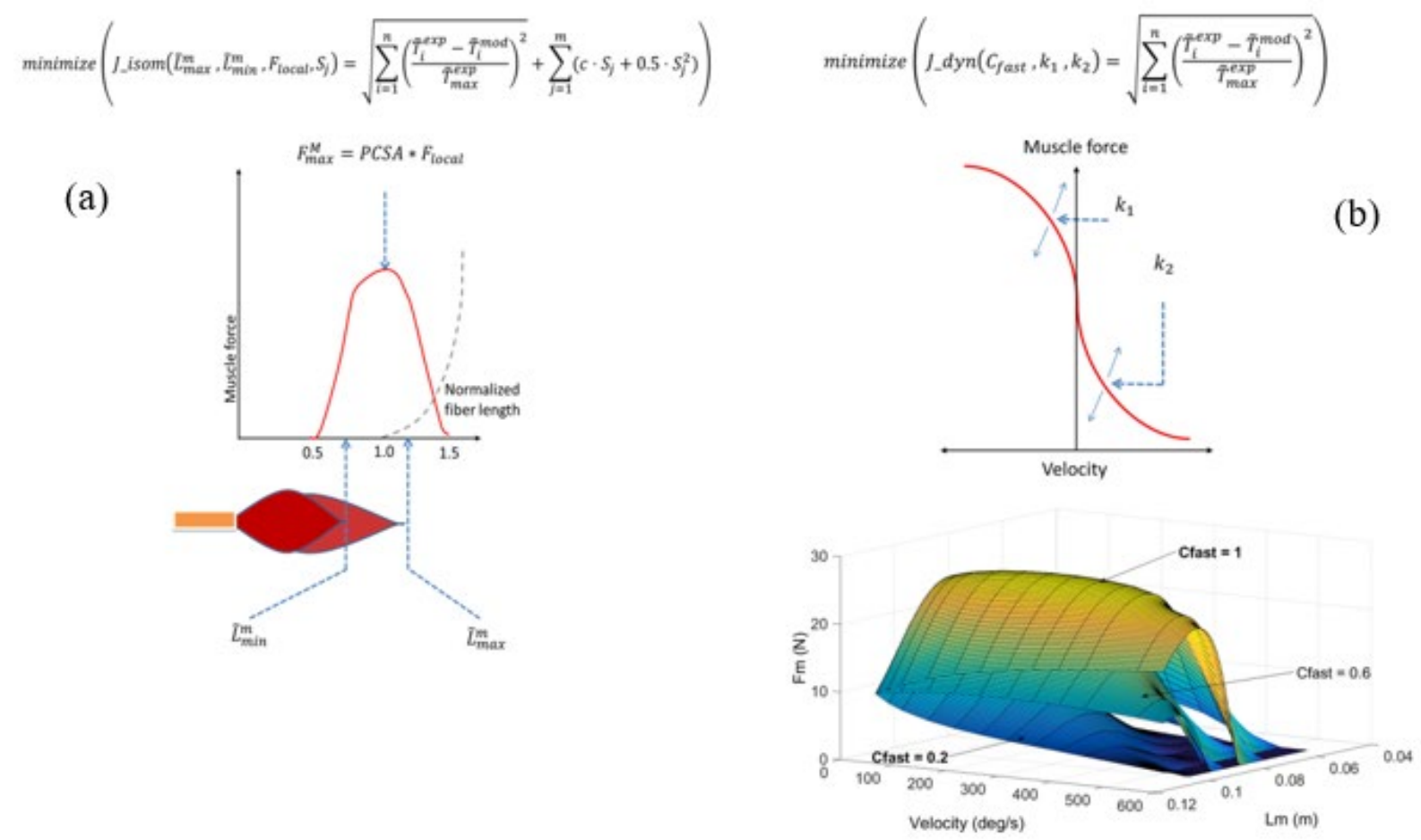

Figure 2. Visual design variable interpretation aid.

\section{Isometric Optimisation (isom-opt)}

The first optimisation problem is based on the method presented by Garner and Pandy [20], concerning only the experimentally measured isometric joint torques. The objective function is to minimise the normalised root squared difference between the measured joint torque and the model-predicted torque, by adjusting the generic force-length relation of the muscle model. We applied the gradient-based Sequential Quadratic Programming (SQP) algorithm SNOPT (Sparse Nonlinear OPTimizer) by Gill et al. [35] with default settings in combination with an elastic programming technique formulation with slack variables, based on Svanbergs work on the Method of Moving Asymptotes (MMA), see Svanberg [36]. The optimisation problems were coded in Python (Python Software Foundation) using the open-source software package PyOpt [37].

Objective function:

$\min \left(J_{\text {isom }}\left(\tilde{L}_{\text {max }}^{m}, \tilde{L}_{\text {min }}^{m}, F_{\text {local }}, S_{j}\right)=\sqrt{\sum_{i=1}^{n}\left(\frac{\tilde{T}_{i}^{\text {exp }}-\tilde{T}_{i}^{\text {mod }}}{\tilde{T}_{\text {max }}^{\text {exp }}}\right)^{2}}+\sum_{j=1}^{m}\left(c \cdot S_{j}+0.5 \cdot S_{j}^{2}\right)\right)$

Constraints:

$\frac{L t_{\min }+\delta}{\delta} \leq \frac{L t_{s}}{\delta}+S \quad$ (Minimum tendon length) 
$\frac{L t_{s}}{\delta}-S \leq \frac{L t_{\max }-\delta}{\delta} \quad$ (Maximum tendon length)

$0.1 \leq \tilde{L}_{\max }^{m}-\tilde{L}_{\min }^{m} \quad$ (Minimum difference between normalised fiber lengths)

The first term of the objective function (V), is summing across the number of joint torques at the different velocities $(n)$, while the second term of the objective function formulation is the summation that accounts for penalisation of the slack variables, $S_{j} \in[0 ; \infty[$, whenever different from the desired zero values across all included muscle-tendon parameters $(m)$. The slack variables in the constraint equations (VI)-(VII) avoid unrealistic solutions during the optimisation and were found to be more robust than SNOPT's own elastic programming capabilities. In agreement with recommendations of Svanberg [38], all of the non-linear constraints (VI)-(VII) were suitably normalised, and the penalisation constant $c$ is set to 10 , both serving the purpose of proper scaling between the objective function and the non-linear constraints, but likewise found to suffice for pushing all of the slack variables to the desired zero values. With the exception of all slack variables, design variable gradients are estimated using finite differences with a step size, $\Delta=5 \times 10-5$, found appropriate by experience. $\widetilde{\mathrm{T}}_{i}{ }^{\exp }$ and $\widetilde{\mathrm{T}}_{i}^{\text {mod }}$ are respectively the experimental and model-predicted net joint torque for the i'th joint position. The difference in joint torques is normalised with respect to the maximal experimental joint torque, $\widetilde{\mathrm{T}}_{\max }^{\exp }$. In addition to the aforementioned slack variables, three design variables (Figure 2a) were chosen for each muscle: a local strength factor $\left(\mathrm{F}_{\text {local }}\right)$, and two normalised fiber lengths; one shorter $\left(\tilde{L}_{\text {min }}^{m}\right)$ and one longer $\left(\tilde{L}_{\max }^{m}\right)$ than the optimal fiber length, derived as presented by Garner and Pandy [20]. The local strength factor was assigned to each muscle group, i.e., extensors/flexors, so that the relative strength between the muscles was maintained. Muscle branches of the same anatomical muscle, were assumed to be similar and hence the same design variables were assigned to all branches. A total of 25 lower extremity muscles were involved in isomopt resulting in 55 design variables and 136 slack variables.

The constraints (VI) and (VII) prevent non-physiological tendon lengths, requiring $L_{t s}$ to stay within the AMS-specific minimum and maximum values, $\mathrm{Lt}_{\text {min }}$ and $\mathrm{Lt}_{\max }$, by a small margin, $\delta=25 \Delta$, found appropriate by experience and considering the finite difference step size $(\Delta)$. A small margin as opposed to the specific limit values within the AMS was found necessary to provide consistent gradient information for SNOPT.

The constraint (VIII) ensures that $\tilde{\mathrm{L}}_{\max }^{\mathrm{m}}$ is larger than $\tilde{\mathrm{L}}_{\min }^{\mathrm{m}}$ in order to avoid negative fiber lengths. The lower and upper bounds for $\tilde{\mathrm{L}}_{\min }^{\mathrm{m}}$ and $\tilde{\mathrm{L}}_{\max }^{\mathrm{m}}$ were based on the reported physiological operating range of a muscle $[9,20]$. Design variable bounds and initial values appear in Table 2.

Table 2. initial values, lower and upper bounds for the isom-opt design and slack variables

\begin{tabular}{cccc}
\hline & Lower bounds & Upper bounds & Initial values \\
\hline$\tilde{L}_{\text {min }}^{m}$ & 0.1 & 0.8 & 0.5 \\
$\tilde{L}_{\text {max }}^{m}$ & 0.7 & 1.6 & 1.2 \\
$F_{\text {local }}$ & 0.1 & 10 & 1 \\
$F c_{\text {fast }}$ & 0 & 1 & 0.4 \\
$S$ & 0 & $\infty$ & 0 \\
\hline
\end{tabular}




\section{Isovelocity Optimisation (dyn-opt)}

A similar setup was used for the second optimisation problem (dyn-opt) and was based on the results off the previous optimisation (isom-opt). However, different design variables (Figure $2 \mathrm{~b}$ ) concerning the force-velocity relation were implemented. These design variables consisted of the proportion of fast twitch fibers $\left(C_{\text {fast }}\right)$, and two force-velocity curve shape parameters $\left(k_{1}\right)$ and $\left(k_{2}\right)$ were included as presented in equations (II) and (III). These variables were selected partly based on what was possible to adjust in AMS related to the force-velocity relation of the muscle model and partly in relation to the muscles physiology. Once more, the objective function minimises the normalised root squared difference between the experimental and modeled net joint torques. This second optimisation problem is unconstrained except for side constraints on the design variables, for which reason slack variables are superfluous. Initial values and lower and upper values for the 18 design variables were based on the default settings in AMS and appears in Table 3.

Objective function:

$\min \left(J_{d y n}\left(C_{f a s t}, k_{1}, k_{2}\right)=\sqrt{\sum_{i=1}^{n}\left(\frac{\tilde{T}_{i}^{\text {exp }}-\tilde{T}_{i}^{\text {mod }}}{\tilde{T}_{\max }^{\text {exp }}}\right)^{2}}\right)$

The lower and upper bounds for $C_{\text {fast }}, k_{1}$ and $k_{2}$ match the standard AMS defined limits and appear in Table 3.

Table 3. initial values, lower and upper bounds for the dyn-opt design variables

\begin{tabular}{cccc}
\hline & Lower bounds & Upper bounds & Initial values \\
\hline$F c_{\text {fast }}$ & 0 & 1 & 0.4 \\
$k_{1}$ & 0 & 10 & 10 \\
$k_{2}$ & 0 & 10 & 0 \\
\hline
\end{tabular}

The optimised model joint torque predictions will be compared to the experimental joint torques and the joint torques predicted by a reference scaled model based on the standard AMS length-mass-fat scaling law.

\section{RESULTS}

The isom-opt converged after approximately 300 iterations on a non-parallelized CPU (Intel Core i7-3970X CPU 3,5G Hz), decreasing the objective function value from $J_{\text {isom }}=2.49$ to $J_{\text {isom }}=0.21$. The smaller dyn-opt problem converged after approximately 200 iterations, decreasing the objective function value from $J_{d y n}=2.81$ to $J_{d y n}=2.17$. The differences between the standard AMS-scaled reference model (ref-model) and the subject-specific isom-opt optimised model, compared to experimental isometric joint torque data is shown in Figure 3. The muscle tendon parameters as a result of isom-opt are shown in in Figure 4. 

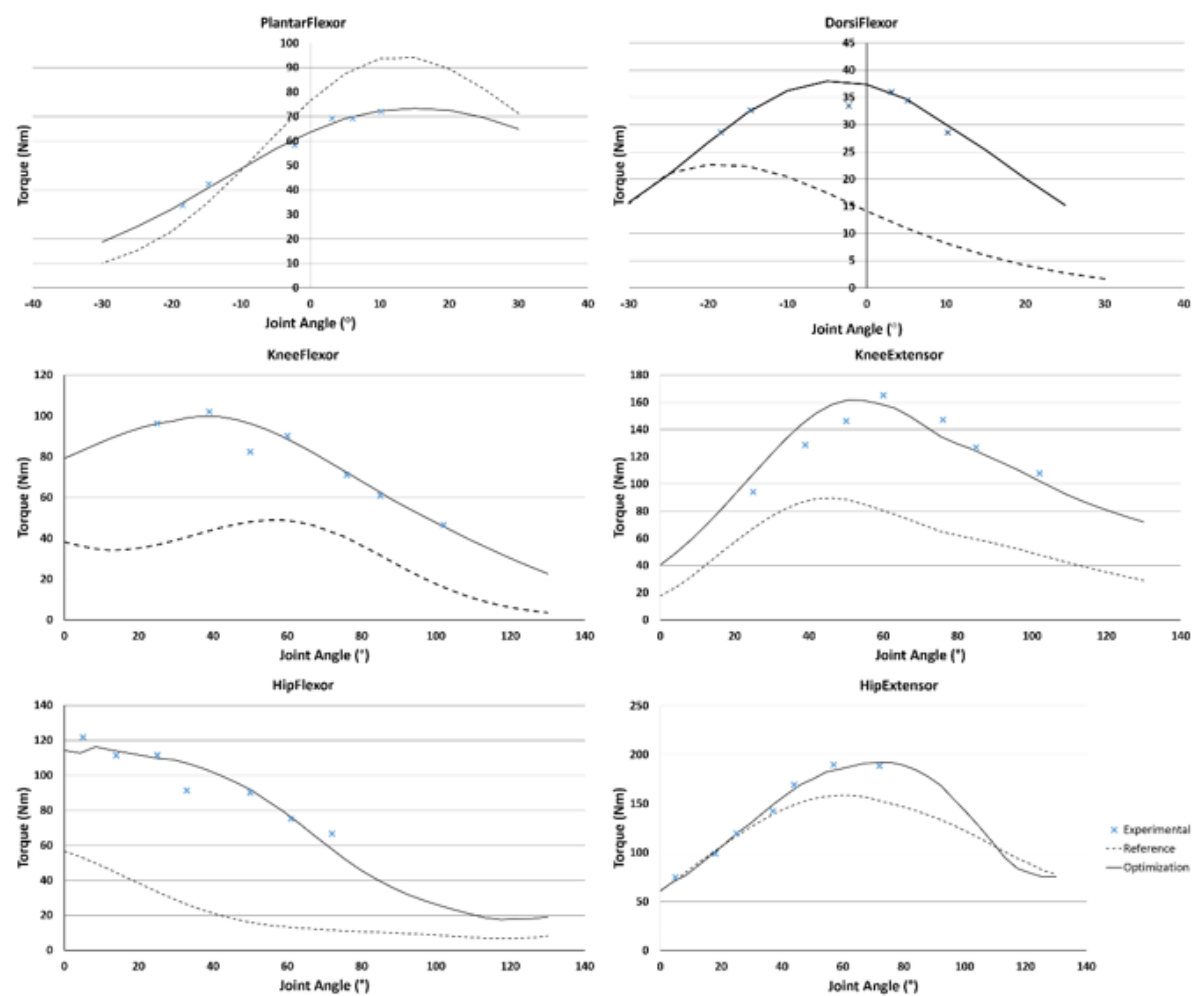

Figure 3. Isometric joint-torque comparison of the experimental (Experimental) values and the predictions of isom-opt (Optimisation) and ref-model (Reference).

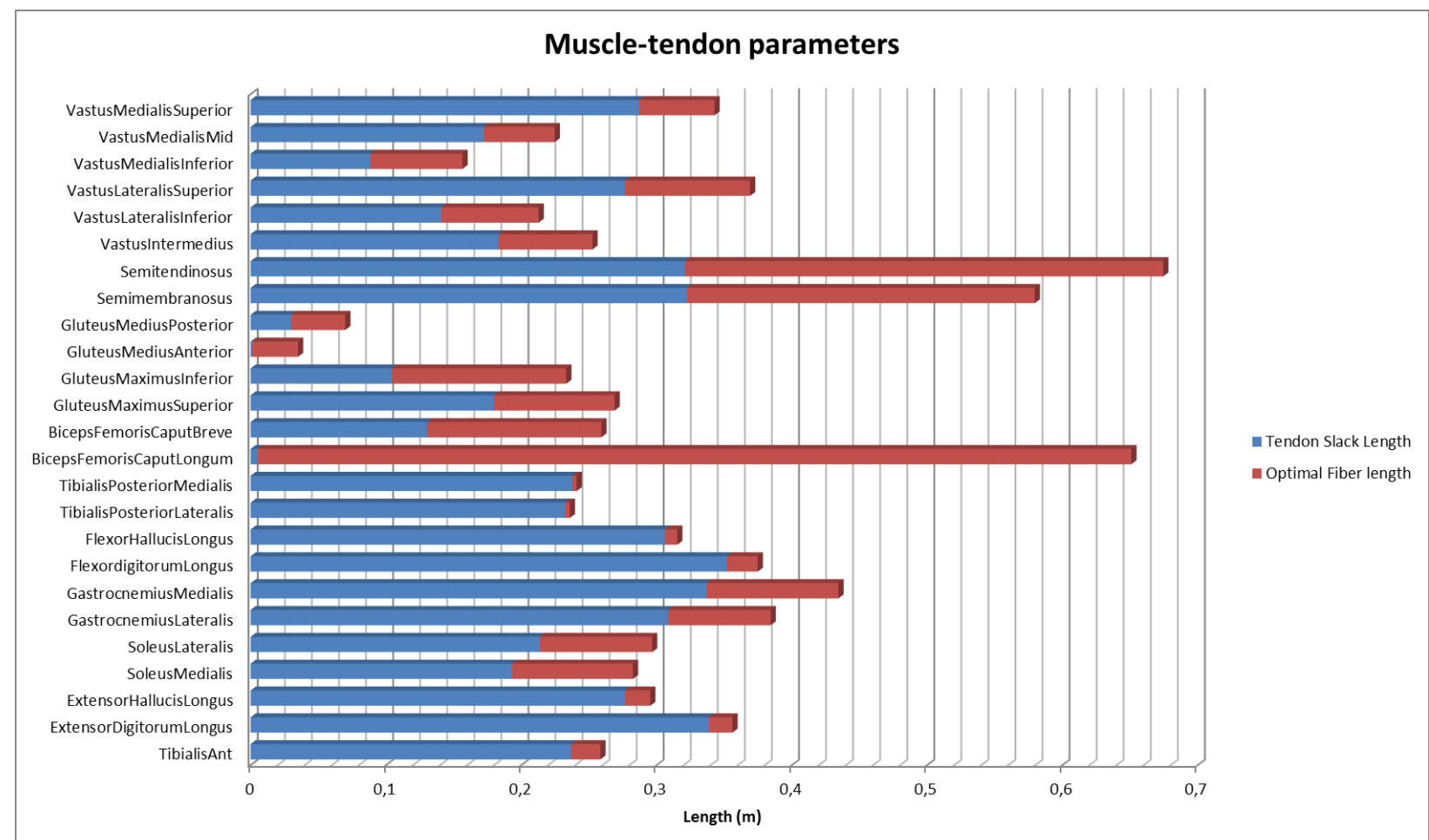

Figure 4. Optimal fiber length and tendon slack length of the included muscle-tendon units in the optimisation. 
The normalised root mean square difference $\left(R M S=\sqrt{\left(\frac{\sum\left(\frac{\left(T_{\text {mod }}-T_{\text {exp }}\right)^{2}}{T_{\text {max }}}\right)}{n}\right)} \times\right.$

$100 \%$, where $n$ is the number of torque measurements) between the experimental and the model-predicted joint torque values were reduced for the optimised model when compared to the reference model (Table 4).

Table 4. Root mean squared difference between the experimental and model predicted isometric, concentric and eccentric joint torques normalised to the number of measurements $(n)$

\begin{tabular}{|c|c|c|c|c|c|c|c|c|}
\hline \multirow[t]{2}{*}{ Joint Torque } & \multicolumn{2}{|c|}{$\begin{array}{l}\text { RMS (\%) } \\
\text { Modelled and } \\
\text { experimental } \\
\text { Isometric }\end{array}$} & \multicolumn{3}{|c|}{$\begin{array}{c}\text { RMS (\%) } \\
\text { Modelled and experimental } \\
\text { concentric }\end{array}$} & \multicolumn{3}{|c|}{$\begin{array}{c}\text { RMS (\%) } \\
\text { Modelled and experimental } \\
\text { eccentric }\end{array}$} \\
\hline & Ref & $\begin{array}{c}\text { Isom- } \\
\text { opt }\end{array}$ & Ref & $\begin{array}{c}\text { Isom- } \\
\text { opt }\end{array}$ & Dyn-opt & Ref & Isom-opt & Dyn-opt \\
\hline Ankle Dorsi & 46.6 & 4.9 & 12.6 & 7.6 & 4.5 & 31.6 & 3.9 & 3.4 \\
\hline Ankle Plantar & 14.8 & 1.8 & 12.1 & 31.1 & 25.3 & 33.1 & 27.2 & 27.5 \\
\hline Knee Flexor & 58.3 & 5.2 & 51.0 & 26.6 & 14.7 & 54.5 & 16.9 & 14.5 \\
\hline Knee Extensor & 38.2 & 7.1 & 20.6 & 16.5 & 18.1 & 49.6 & 56.1 & 53.2 \\
\hline Hip Flexor & 55.5 & 6.5 & 34.5 & 28.3 & 34.4 & 14.6 & 15.6 & 10.4 \\
\hline Hip Extensor & 10.3 & 2.2 & 33.4 & 23.9 & 26.8 & 50.6 & 29.8 & 31.0 \\
\hline Mean of above & 37.3 & 4.6 & 27.4 & 22.3 & 20.6 & 39.0 & 24.9 & 23.3 \\
\hline
\end{tabular}

On average, an improved predicted concentric isovelocity joint torque compared with both the ref-model and isom-opt (Figure 5) was achieved by dyn-opt. The average root mean squared (RMS) difference was decreased from $27.4 \%$ (ref-model) and $22.3 \%$ (isom-opt) to $20.6 \%$ (dyn-opt). For the ankle plantar flexor joint torque, the RMS difference was larger than the ref-model whilst, for the hip flexor joint torque, no difference between the three models was observed (Table 4).

Predictions of eccentric isovelocity joint torque (Table 4, Figure 6) were better using the dyn-opt $(39.0 \%)$ compared to both the ref-model $(24.9 \%)$ and isom-opt $(23.3 \%)$. 

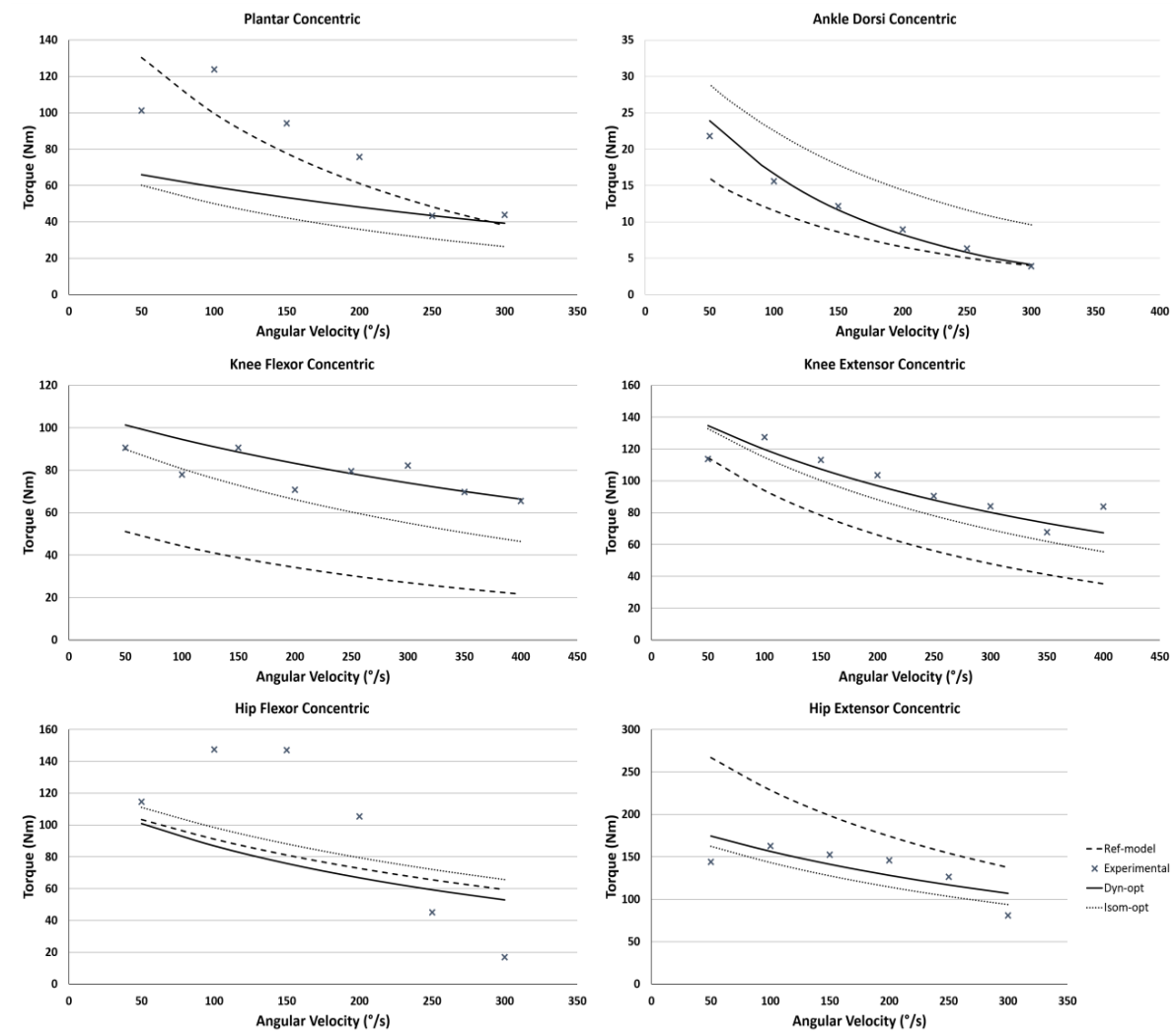

Figure 5. Concentric isovelocity joint-torque comparison of the experimental (Experimental) values, the predicted from isom-opt, the predicted from the isometric and dyn-opt and ref-model.
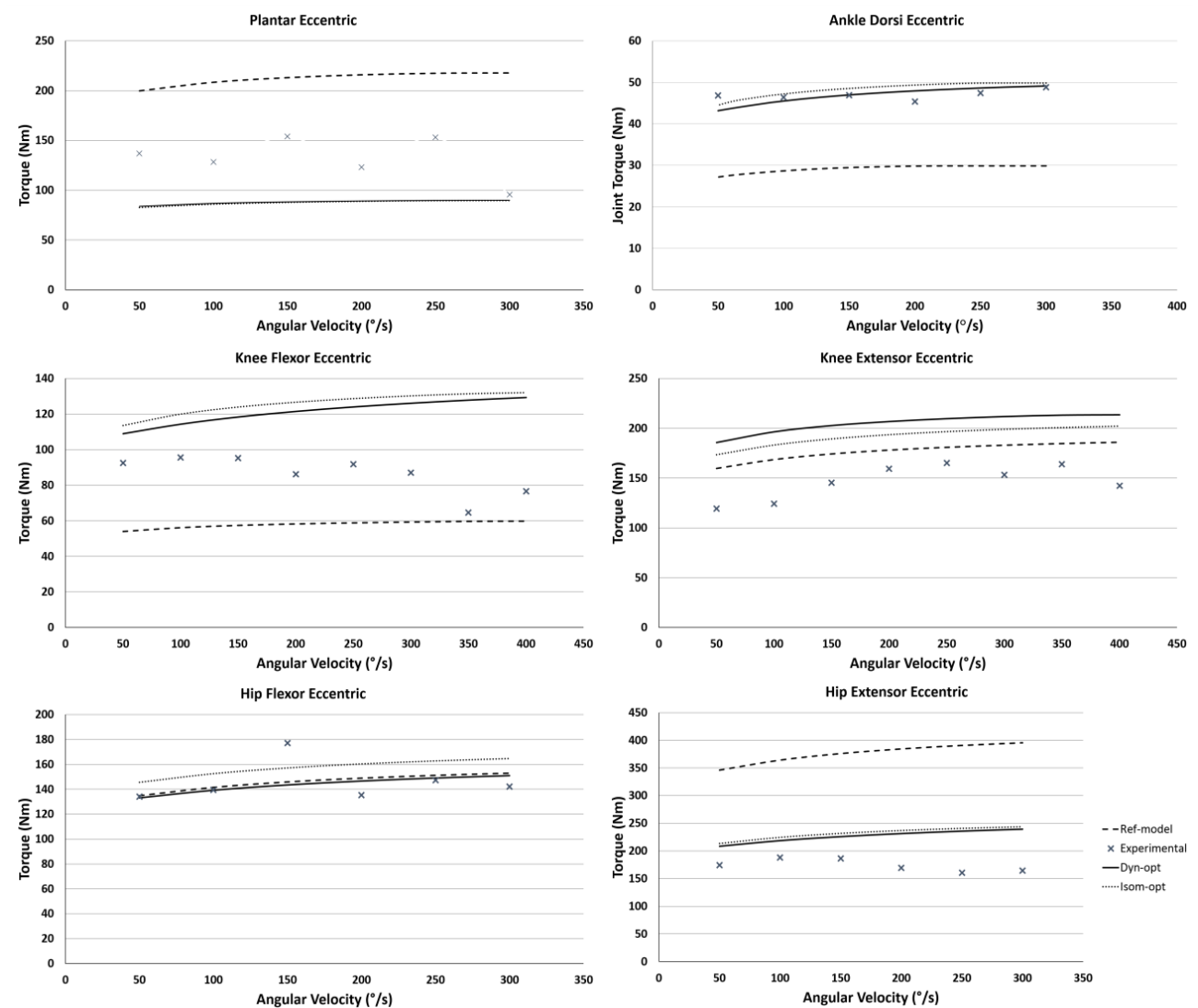

Figure 6. Eccentric isovelocity joint-torque comparison of the experimental measurements, the predicted torques from isom-opt, the predicted torques from the isometric and dyn-opt and the ref-model. 


\section{DISCUSSION}

The optimisation problem was formulated in two stages; an isometric (isom-opt) and an isovelocity (dyn-opt) part. This was found to be advantageous given the nature of the experimental data where isovelocity data is more prone to noise and submaximal effort from the participant than the isometric measurements. Furthermore, at high angular velocities a larger portion of the range of motion is used in order to accelerate and decelerate the joint to isovelocity. This leads to less available data at higher velocities and is combined with more noise.

A large decrease in the average RMS for all joint actions between the ref-model $(112.4 \%)$ and isom-opt $(15.5 \%)$ was observed for the predicted isometric joint torques. For the concentric and eccentric joint torques, the average RMS difference was decreased from ref-model to isom-opt and even further when implementing the dyn-opt (Table 4), thereby consistently improving the model by consecutive optimisations and making the model a better representation of the participant's joint torque profile. However, as it can be observed in Figures 3-6 and Table 4, that only small improvements were observed between the isom-opt and dyn-opt. This is especially apparent for the eccentric joint torques where a mean improvement of 42.1 percentage points was achieved. It is relevant to note that these small improvements are the product of a relatively large additional effort in both collecting experimental data and then optimisation, and that a good estimate can be made using the less labour-intensive protocol of collecting and optimising only to isometric measurements. This is especially pertinent when simulating slow velocity movements. For simulating high velocity movements, it is apparent that it is still advantageous to estimate the maximal joint torque using dynamic torque measurements. An advantage of establishing a set of subject-specific MTP is that it gives one unique set of parameters that can be used in different models and simulations without the need for establishing them again. This is opposite to EMG driven models [39] which require experimental data from the movement that should be modeled and a new set of parameters needs to be established each time.

The isom-opt adjusted the MTP in the model. It was observed that after the isom-opt some of the fiber lengths deviated significantly from literature values [2, 40] and may not be physiologically reasonable. In order to avoid unrealistic fiber lengths, narrower bounds of the design variables could be implemented. This was not done in the present study to avoid manual tuning of the result as much as possible. Furthermore, the implemented Hill-muscle model is a phenomenological model which does not represent a human muscle in all aspects but rather mimics its idealised mechanics. Hence, differences can be expected between in vivo/vitro measurements and modelled values [41]. In the present study, we have taken a hybrid approach to the model and based some information on literature, some on experimental information and some on a combination of the two. The optimisation method favored the isometric measurements due to less noise and motion artifact. However we observed smaller measured ankle plantar flexor isometric joint torques (Figure 3) than concentric joint torques (Figure 5), which could indicate that the participant did not reach their maximum during the isometric contractions.

The dyn-opt was not able to achieve similar joint torque profiles to the experiment despite the design variables converging close to their bounds. This may be partly due to the nature of isovelocity measurements which are prone to errors resulting from joint misalignment, noise and soft tissue deformation [34]. In this study particular care was taken to minimise free play in the system using strapping and to align the joint and cranks centre's of rotation under load, and therefore other 
mechanisms must be involved. Furthermore, performing maximal eccentric contractions can be unpleasant for the participant and can therefore result in submaximal measurements and a poor assumption of maximal isometric and concentric contractions as is assumed in the model (Figure 6), especially given that the Hill-type model always predicts higher eccentric than concentric strength of the muscle [9]. It could be argued that the combination of eccentric design variables and the inherent nature of the muscle model do not allow enough flexibility for model tuning to the experimental data. Given the noisy character of the dynamic experimental data, they cannot be regarded as a gold standard, and balancing the confidence in data with the trust in the phenomenological muscle model and constraints on parameter variations requires difficult choices.

The subject included in this trial deviates from the general population, displaying in general a somewhat lower peak joint torque than what can be observed in literature. The ankle plantar flexor maximal isometric joint torque (Figure 3) was lower than what was achieved during the concentric measurements (Figure 5), which may indicate that the participant did not reach his maximum during the isometric contractions. This explanation is more likely than measurement errors due to joint misalignment, noise and soft tissue deformation. Regardless of the reason, lowerthan-expected isovelocity strengths do not influence the isovelocity results significantly, because the optimized design variables specific to dynamic contractions are close to the imposed bounds.

While measuring the plantar flexor joint torque the knee joint angle was kept constant and similar for the knee and hip measurements where the hip and knee angle respectively were maintained. By keeping these angles constant during the measurements we have not explicitly investigated how the biarticular muscles would contribute to the joint torque [19], however, since the musculoskeletal model contains muscles rather than joint actuators, the biarticularity is supposedly already taken into account. Furthermore, the biarticular muscles in this optimisation were included in the optimisation of all the spanning joints and therefore would be affected equally.

This study implemented the ankle, knee and hip joint torques for flexion and extension under both isometric and isovelocity conditions. The model implemented the whole lower extremity where some of the muscles were biarticular thereby creating a certain dependence between the joint torques. Hence the results should be viewed as a whole and cannot be directly compared to those from other studies which have only implemented single joint measures and for a limited number of muscles [15, 21].

Maximal joint torque measurements were collected in order to determine the associated MTP. This method is most viable when modelling athletes who are used to perform maximally and would not be suitable for subjects such as patients since they might not be able to perform maximal contractions. The participant used in this study displayed strength characteristics of the hip, knee and ankle flexors/extensors that are comparable with what have been reported in literature for athletes. The MTP of this study cannot be generalised for a population but is specific to this one included subject. Optimization for additional subjects would provide additional information on inter-subject strength variability and on the robustness and validity of the numerical method. This is left to future work.

Prediction ability of the optimized model for submaximal activities is a subject of much practical interest. Given the strength improvement, the model should be able to predict better than the scaled reference model, but this cannot be verified with the experimental methods employed in this paper and will be the scope for future studies. 
The gradient-based optimiser SNOPT was applied in this study. SNOPT has been found to work well and provide a significant design criteria improvement compared to reference-scaled values. Obtained results may nevertheless be suboptimal in the strict sense since they have not been identified with a global optimisation, but dedicated global optimisers require many more iterations in order to reasonably uncover the whole design space to identify the global optimum. This would increase the optimisation convergence time significantly and make the process infeasible using normal computers. accurate.

\section{CONCLUSION}

MTP of a musculoskeletal model of the whole lower extremity were scaled based on individually obtained measured torque profiles using a two-fold optimisation procedure with the goal of creating a subject-specific model. Both an isometric only and a combined isometric and dynamic method of optimisation resulted in large improvements in the prediction of joint torque profiles when compared to those predicted using the standard parameters of the AnyBody Modeling System. Depending on the purpose of the model, it should be considered whether an isometric only approach to optimisation could offer adequate accuracy, taking into account the extra measurement and analysis demands of a combined isometric and dynamic optimisation approach. 


\section{REFERENCES}

[1] Arnold EM, Ward SR, Lieber RL, et al. A Model of the Lower Limb for Analysis of Human Movement. Ann Biomed Eng 2010; 38: 269-279.

[2] Klein Horsman MD, Koopman HFJM, van der Helm FCT, et al. Morphological muscle and joint parameters for musculoskeletal modelling of the lower extremity. Clin Biomech 2007; 22: 239-247.

[3] Holmberg LJ and Lund AM. A musculoskeletal full-body simulation of cross-country skiing. J Sports Eng Technol 2008; 222: 11-22.

[4] Wu JZ, Chiou SS and Pan CS. Analysis of musculoskeletal loadings in lower limbs during stilts walking in occupational activity. Ann Biomed Eng 2009; 37: 11771189.

[5] Fregly BJ, Reinbolt JA, Rooney $\mathrm{KL}$, et al. Design of patient-specific gait modifications for knee osteoarthritis rhabilitation. IEEE Trans Biomed Eng 2007; 54: 1687-1695.

[6] Rasmussen J, Holmberg LJ, Sørensen K, et al. Performance optimization by musculoskeletal simulation. Move Sport Sci 2012; 1: 73-83.

[7] Rasmussen J, Kwan MMS, Andersen MS, et al. Analysis of segment energy transfer using musculoskeletal models in a high speed badminton stroke. In: Anonymous , 24/02-10.

[8] Hill AV. The heat of shortening and dynamics constants of muscles. Proc Royal Soc 1938; 126: 136-195.

[9] Zajac FE. Muscle and tendon: properties, models, scaling, and application to biomechanics and motor control. Crit Rev Biomed Eng 1989; 17: 359-411.

[10] Redl C, Gfoehler M and Pandy MG. Sensitivity of muscle force estimates to variations in muscle-tendon properties. Hum Mov Sci 2007; 26: 306-319.

[11] Scovil CY and Ronsky JL. Sensitivity of a Hill-based muscle model to perturbations in model parameters. J Biomech 2006; 39: 2055-2063.

[12] Ward SR, Smallwood LH and Lieber RL. Scaling of human lower extremity muscle architecture to skeletal dimensions. In: XXth ISB Congress - ASB 29th Annual MeetingAnonymous, Cleveland, Ohio, United States of America, July 31 - August 5, pp.502.

[13] Heinen F, Lund ME, Rasmussen J, et al. Muscle-tendon unit scaling methods of Hill-type musculoskeletal models: An overview. Proc Inst Mech Eng H 2016.

[14] Winby CR, Lloyd DG and Kirk TB. Evaluation of different analytical methods for subject-specific scaling of musculotendon parameters. J Biomech 2008; 41: 16821688.

[15] Van Campen A, Pipeleers G, De Groote F, et al. A new method for estimating subject-specific muscle-tendon parameters of the knee joint actuators: a simulation study. Int J Numer Meth Biomed Eng 2014; 30: 969-987.

[16] King MA and Yeadon MR. Determining subject specific torque parameters for use in a torque driven simulation model of dynamic jumping. J APPL BIOMECH 2002; 18: $207-217$.

[17] King MA, Wilson $\mathrm{C}$ and Yeadon MR. Evaluation of a Torque-Driven Model of Jumping for Height. J APPL BIOMECH 2006; 22: 264-274. 
[18] Yeadon MR, King MA and Wilson C. Modelling the maximum voluntary joint torque/angular velocity relationship in human movement. J Biomech 2004: 476482.

[19] Lewis MGC, King MA, Yeadon MR, et al. Are Joint Torque Models Limited by an Assumption of Monoarticularity?. J APPL BIOMECH 2012; 28: 520-529.

[20] Garner BA and Pandy MG. Estimation of Musculotendon Properties in the Human Upper Limb. Ann Biomed Eng 2003; 31: 207-220.

[21] Hasson C, J. and Caldwell G, E. Effects of Age on Mechanical Properties of Dorsiflexor and Plantarflexor Muscles. Ann Biomed Eng 2012; 40: 1088-1101.

[22] Wu W, Lee PVS, Bryant AL, et al. Subject-specific musculoskeletal modeling in the evaluation of shoulder muscle and joint function. Journal of Biomechanics 2016; 49: 3626-3634.

[23] d'Souza S, Brückner B, Rasmussen J, et al. Development of age and gender based strength scaled equations for use in simulation models 2011.

[24] Schwartz FP, Bottaro M, Celes RS, et al. The influence of velocity overshoot movement artifact on isokinetic knee extension tests. J Sports Sci Med 2010; 9: 140-146.

[25] Carbone V, Fluit R, Pellikaan P, et al. TLEM 2.0 - A comprehensive musculoskeletal geometry dataset for subject-specific modeling of lower extremity. J Biomech 2015; 48: 734-734-741.

[26] Daxner T. Simulation von Beuge-und Streckbewegungen des menschlichen Knies mit DADS, Ph.D. dissertation, Institut für Mechanik der Technischen Universität Wien, A-4810 Gmunden Hochmüllergasse 22, Wien Matr.Nr.: 8825624, 1997.

[27] Otten E. Morphometrics and force-length relations of skeletal muscles. In: Anonymous International Series on Biomechanics (ISB) Biomechanics IX-A, 1985, p.27.

[28] Gföhler M, Angeli T, Eberharter T, et al. Dynamic simulation of cycling powered by lower extremity muscles activated by functional electrical stimulation. In: XIlth International Biomechanics SeminarAnonymous , pp.101-122.

[29] Kaufman KR, An K and Chao EY. Incorporation of muscle architecture into the muscle length-tension relationship. J Biomech 1989; 22: 943-948.

[30] Marra MA, Vanheule V, Fluit R, et al. A Subject-Specific Musculoskeletal Modeling Framework to Predict In Vivo Mechanics of Total Knee Arthroplasty. J Biomech Eng 2015; 137: 020904-020904.

[31] de Zee M, Dalstra M, Cattaneo PM, et al. Validation of a musculo-skeletal model of the mandible and its application to mandibular distraction osteogenesis. J Biomech 2007; 40: 1192-1201.

[32] Rasmussen J, Damsgaard $M$ and Voigt $M$. Muscle recruitment by the $\mathrm{min} / \mathrm{max}$ criterion - a comparative numerical study. J Biomech 2001; 34: 409-415.

[33] Rasmussen J, de Zee M, Damsgaard M, et al. A General Method for Scaling Musculo-Skeletal Models. Int Symp Comput Simul Biomech 2005; Cleveland, Ohio, United States. 
[34] A. Van Campen, F. De Groote, I. Jonkers, et al. An Extended Dynamometer Setup to Improve the Accuracy of Knee Joint Moment Assessment. IEEE Trans Biomed Eng 2013; 60: 1202-1208.

[35] Gill PE, Murray W and Saunders MA. SNOPT: An SQP algorithm for large-scale constrained optimization. SIAM J OPTIMIZ 2002; 12: 979-1006.

[36] Svanberg K. THE METHOD OF MOVING ASYMPTOTES - A NEW METHOD FOR STRUCTURAL OPTIMIZATION. Int J Numer Methods Eng 1987; 24: 359373.

[37] Perez RE, Jansen PW and Martins JRRA. pyOpt: A Python-Based ObjectOriented Framework for Nonlinear Constrained Optimization. Struct Muttidiscip Opt 2012; 45: 101-118.

[38] Svanberg K. Some modelling aspects for the fortran implementation of MMA.

[39] Lloyd DG and Besier TF. An EMG-driven musculoskeletal model to estimate muscle forces and knee joint moments in vivo. Journal of Biomechanics 2003: 765-776.

[40] Friederich JA and Brand RA. Muscle Fiber Architecture in the Human Lower Limb. J Biomech 1990; 23: 91-95.

[41] van den Bogert AJ, Gerritsen KGM and Cole GK. Human muscle modelling from a user's perspective. J Electromyogr Kinesiol 1998; 8: 119-124. 